ARTICLE

\title{
Photoinduced site-selective alkenylation of alkanes and aldehydes with aryl alkenes
}

Hui Cao ${ }^{1,2}$, Yulong Kuang (1) ${ }^{1}$, Xiangcheng Shi ${ }^{1}$ Koi Lin Wong ${ }^{1}$, Boon Beng Tan', Jeric Mun Chung Kwan',

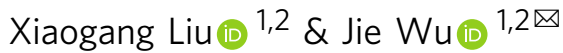

The dehydrogenative alkenylation of $\mathrm{C}-\mathrm{H}$ bonds with alkenes represents an atom- and stepeconomical approach for olefin synthesis and molecular editing. Site-selective alkenylation of alkanes and aldehydes with the $\mathrm{C}-\mathrm{H}$ substrate as the limiting reagent holds significant synthetic value. We herein report a photocatalytic method for the direct alkenylation of alkanes and aldehydes with aryl alkenes in the absence of any external oxidant. A diverse range of commodity feedstocks and pharmaceutical compounds are smoothly alkenylated in useful yields with the $\mathrm{C}-\mathrm{H}$ partner as the limiting reagent. The late-stage alkenylation of complex molecules occurs with high levels of site selectivity for sterically accessible and electron-rich $\mathrm{C}-\mathrm{H}$ bonds. This strategy relies on the synergistic combination of direct hydrogen atom transfer photocatalysis with cobaloxime-mediated hydrogen-evolution cross-coupling, which promises to inspire additional perspectives for selective $\mathrm{C}-\mathrm{H}$ functionalizations in a green manner.

\footnotetext{
${ }^{1}$ Department of Chemistry, National University of Singapore, 3 Science Drive 3, Singapore 117543, Republic of Singapore. ${ }^{2}$ National University of Singapore (Suzhou) Research Institute, 377 Lin Quan Street, Suzhou Industrial Park, Suzhou, Jiangsu 215123, P. R. China. ${ }_{\text {email: chmiie@nus.edu.sg }}$
} 
$\mathrm{O}$

lefins play an essential role in organic synthesis owing to their extremely rich chemistry ${ }^{1}$. The direct alkenylation of simple $\mathrm{C}-\mathrm{H}$ bonds with alkenes is one of the most appealing approaches for the construction and derivatization of complex molecules ${ }^{2,3}$. While transition-metal-catalyzed alkenylations of arenes and heteroarenes have been extensively studied $^{4-6}$, analogous transformations of alkanes and aldehydes remain rare ${ }^{7-20}$. Expanding direct alkenylation to $\mathrm{C}_{\mathrm{sp} 3}-\mathrm{H}$ and $\mathrm{C}_{\mathrm{sp2}}(\mathrm{O})-\mathrm{H}$ bonds is challenging due to the slow metalmediated $\mathrm{C}-\mathrm{H}$ cleavage ${ }^{21}$, the instability of alkyl/acyl metallic intermediates $^{22,23}$, and the selectivity issues arising from the ubiquity of $\mathrm{C}_{\mathrm{sp} 3}-\mathrm{H}$ bonds in organic molecules ${ }^{24}$.

Transition-metal-catalyzed dehydrogenative alkenylation of alkanes and aldehydes through an auxiliary-assisted metal insertion was recently disclosed (Fig. 1a) ${ }^{7-13}$. However, these transformations usually require stoichiometric $\mathrm{Cu}^{\mathrm{II}}$ or $\mathrm{Ag}^{\mathrm{I}}$ salts as oxidants. Moreover, the heteroatom-containing directing groups can undergo intramolecular Michael addition and give cyclized products instead of the desired alkenylated products ${ }^{7-13}$. In 2019, Gevorgyan and coworkers disclosed an elegant radical relay strategy for the alkenylation of alcohols with visible-lightmediated palladium catalysis ${ }^{14}$. Nonetheless, the requirement of a silicon-based auxiliary lowers the atom economy of this strategy, and the substrate scope is limited to alcohols. On the other hand, auxiliary-free dehydrogenative alkenylation offers practical advantages and has attracted substantial interest (Fig. 1b) ${ }^{15-20}$. Although much progress has been achieved, the developed systems usually require stoichiometric peroxides and a large excess of the $\mathrm{C}-\mathrm{H}$ partner (over 40 equivalents of the alkanes), and the substrate scopes are limited to simple hydrocarbons and aromatic aldehydes. In pursuing methods for olefin construction ${ }^{25-27}$ and photomediated $\mathrm{C}-\mathrm{H}$ bond functionalization ${ }^{28-31}$, we aspired to develop an auxiliary-free and oxidant-free strategy for $\mathrm{C}_{\mathrm{sp} 3}-\mathrm{H}$ and $\mathrm{C}_{\mathrm{sp} 2}(\mathrm{O})-\mathrm{H}$ alkenylation with the $\mathrm{C}-\mathrm{H}$ substrate as the limiting reagent (Fig. 1c). Importantly, this would allow the late-stage functionalization of advanced synthetic intermediates and pharmaceutical relevant molecules without de novo synthesis ${ }^{32}$.

In this context, we were inspired by direct hydrogen atom transfer (HAT) photocatalysts that could achieve the straightforward activation of $\mathrm{C}-\mathrm{H}$ bonds ${ }^{33}$. Of particular interest was the

a

Auxiliary-assisted alkenylation:

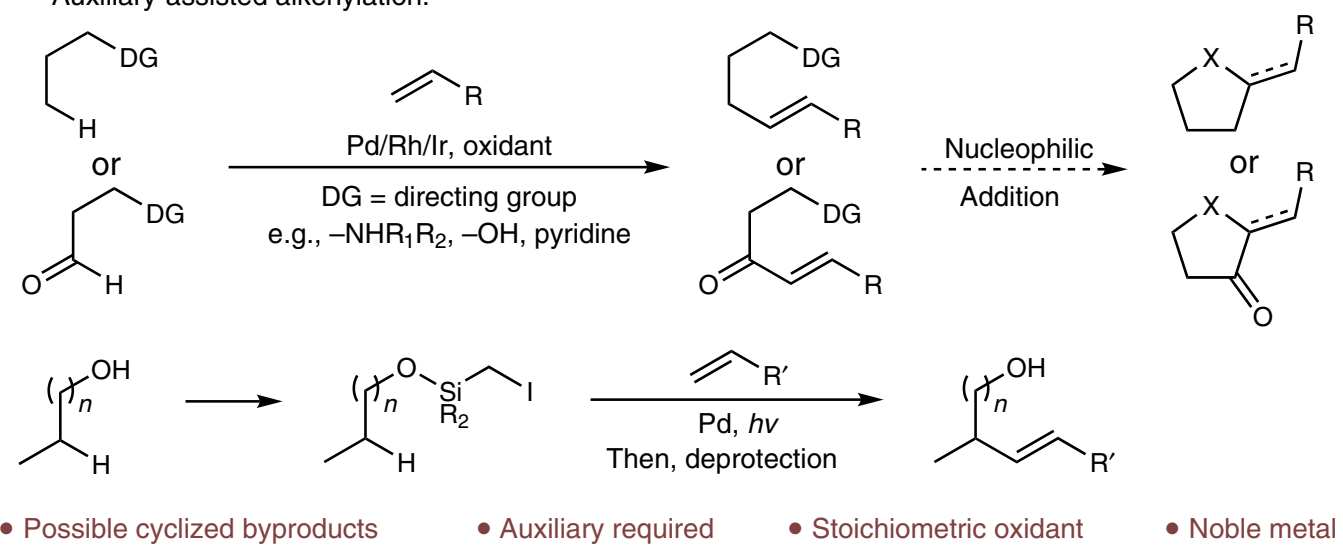

b

Auxiliary-free alkenylation:

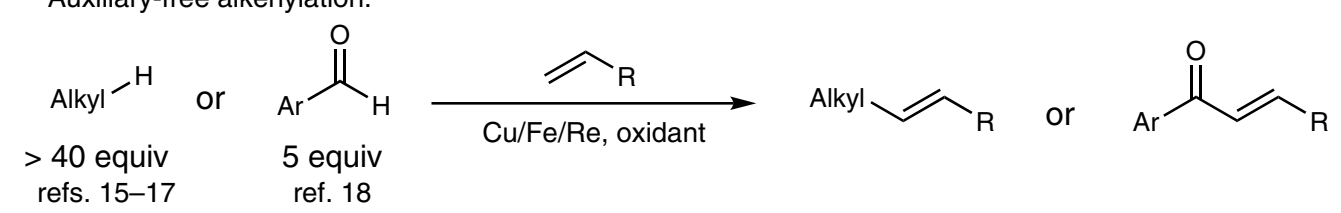

- Large excess of $\mathrm{C}-\mathrm{H}$ partners $\quad$ - Limited substrate scope $\quad$ Stoichiometric oxidant $\quad$ E/Z mixtures

C This work (hydrogen atom transfer catalysis \& cobalt catalysis):

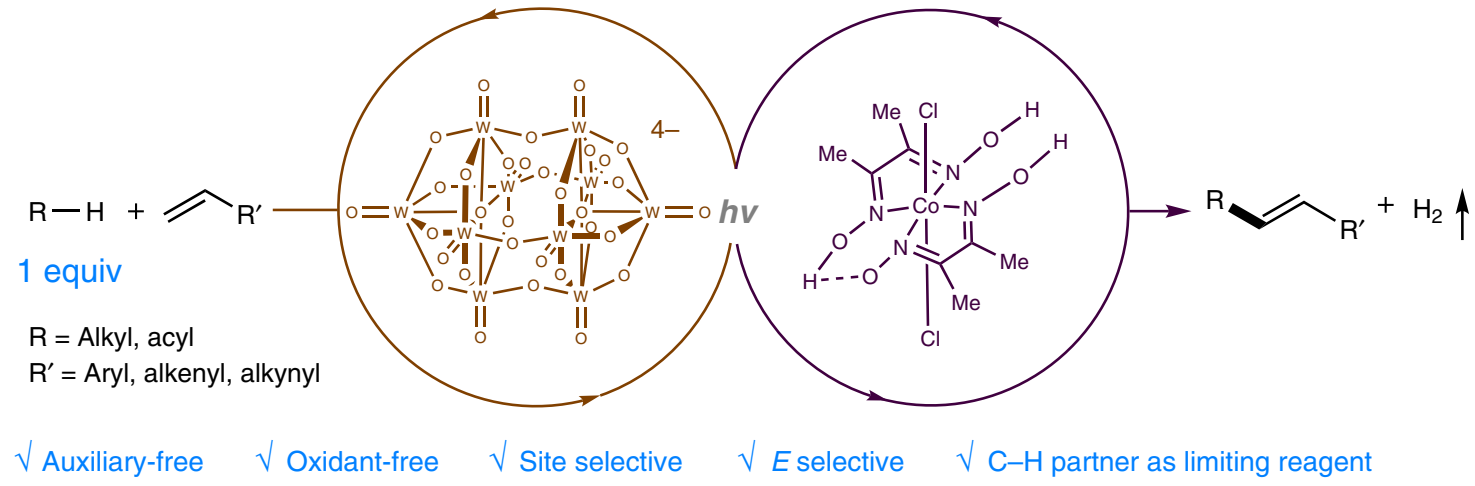

Fig. 1 Strategies for the dehydrogenative alkenylation of alkanes and aldehydes with alkenes. a Auxiliary-assisted alkenylation. b Auxiliary-free alkenylation. c Merging of a hydrogen atom transfer photocatalyst and a cobaloxime catalyst enables site-selective direct alkenylation of $\mathrm{C}-\mathrm{H}$ substrates. 


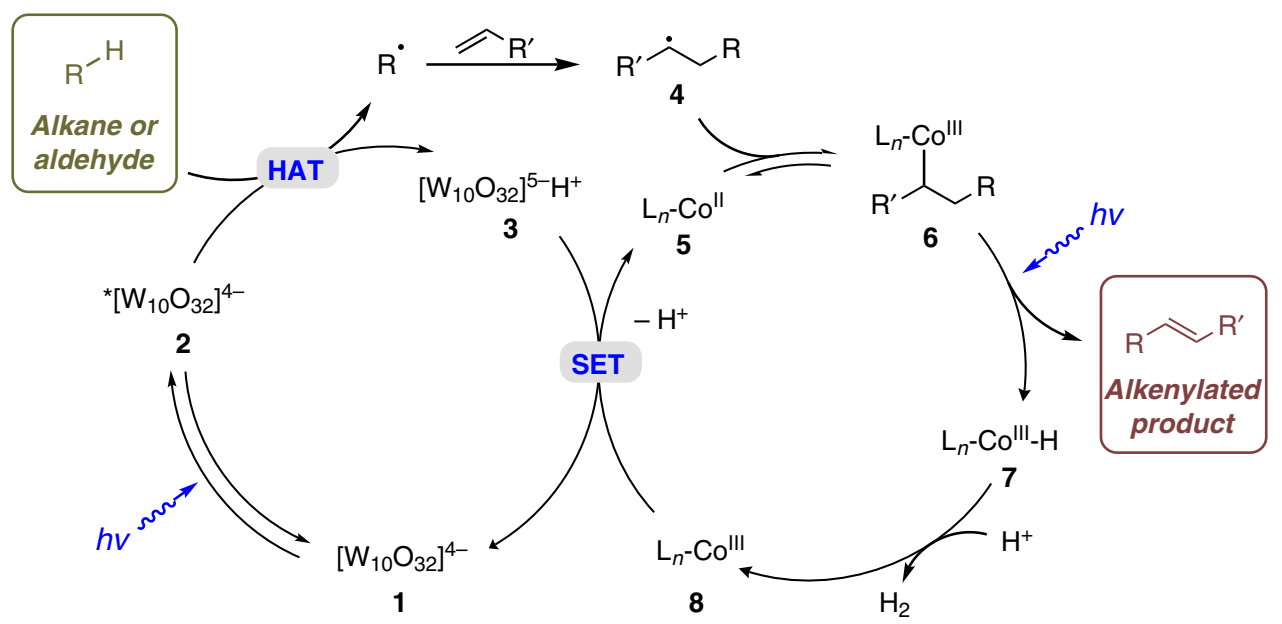

Fig. 2 Proposed mechanism for the dehydrogenative alkenylation of alkanes and aldehydes. The plausible mechanism involves a hydrogen atom transfer process, a single electron transfer process, and cobalt-catalyzed alkene formation and hydrogen evolution.

decatungstate anion $\left(\left[\mathrm{W}_{10} \mathrm{O}_{32}\right]^{4-}\right)$, a polyoxometalate photocatalyst that has been broadly applied in various functionalizations of alkanes and aldehydes ${ }^{34-39}$. On the other hand, photocatalytic dehydrogenative cross-coupling reactions with concomitant hydrogen evolution have recently been developed through cooperative photoredox and cobaloxime catalysis, as pioneered by the $\mathrm{Wu}$ and Lei groups ${ }^{40-44}$. Inspired by these studies, we proposed that the combination of decatungstate anion and a cobaloxime catalyst could enable the direct activation and alkenylation of alkanes and aldehydes using alkenes as the feedstocks. We herein report that by incorporating decatungstate catalysis and cobaloxime catalysis, direct alkenylation of alkanes and aldehydes with aryl alkenes can be achieved in the absence of hydrogen acceptors. This strategy features a broad substrate scope, high C-H site selectivity, excellent $E$ selectivity of the alkene products, and the use of the $\mathrm{C}-\mathrm{H}$ substrate as the limiting reagent. Application of this strategy in late-stage functionalization was demonstrated by the selective alkenylation of natural products and pharmaceutically important molecules.

\section{Results}

Proposed reaction mechanism. A hypothesized catalytic cycle is depicted in Fig. 2. Photoexcitation of decatungstate anion $\mathbf{1}$ would produce triplet excited state 2 after intersystem crossing ${ }^{45}$. Excited $\mathbf{2}$ can abstract hydrogen atoms from the alkane or aldehyde and afford carbon-centered radical $\mathrm{R} \bullet$ and reduced decatungstate $3^{45}$. Subsequent addition of radical $\mathrm{R} \bullet$ to an alkene would furnish radical intermediate 4 , which can be reversibly captured by $\mathrm{Co}^{\mathrm{II}} \mathbf{5}$ to form $\mathrm{Co}^{\mathrm{III}}$ intermediate $6^{46,47}$. Photo-irradiation of the alkyl $\mathrm{Co}^{\mathrm{III}}$ complex would deliver the alkene product along with a $\mathrm{Co}^{\mathrm{III}}-\mathrm{H}$ intermediate via a formal $\beta$-H elimination process $^{46,47}$. $\mathrm{Co}^{\mathrm{III}}-\mathrm{H} 7$ would react with another proton to release $\mathrm{H}_{2}$ and deliver $\mathrm{Co}^{\mathrm{III}} \mathbf{8}^{48-50}$. Finally, a single-electron reduction of $\mathrm{Co}^{\mathrm{III}} \mathbf{8}$ $\left(E_{1 / 2} \mathrm{Co}{ }^{\mathrm{III}} / \mathrm{Co}^{\mathrm{II}}=-0.68 \mathrm{~V} \text { versus } \mathrm{Ag} / \mathrm{Ag}^{+} \text {in } \mathrm{MeCN}\right)^{50,51}$ by reduced decatungstate $3\left(E_{1 / 2}\left[\mathrm{~W}_{10} \mathrm{O}_{32}\right]^{4-} /\left[\mathrm{W}_{10} \mathrm{O}_{32}\right]^{5-}=-0.96\right.$ $\mathrm{V}$ versus $\mathrm{Ag} / \mathrm{Ag}^{+}$in $\left.\mathrm{MeCN}\right)^{52}$ would regenerate both catalysts. With this hypothesis in mind, we investigated the direct alkenylation of alkanes and aldehydes under various conditions.

Reaction optimization. Our investigation began with the alkenylation of cyclooctane with cyclooctane as the limiting reagent and styrene as the alkene partner. After extensive evaluation (Table 1 and Supplementary Tables 1-10), we established that the combination of tetra- $n$-butylammonium decatungstate (TBADT, 9, $4 \mathrm{~mol} \%), \mathrm{Co}(\mathrm{dmgH})(\mathrm{dmgH})_{2} \mathrm{Cl}_{2}(\mathbf{1 0}, 1 \mathrm{~mol} \%)$, and 2,6-lutidine
(L5, $10 \mathrm{~mol} \%)$ in acetonitrile $(0.1 \mathrm{M})$ at $60{ }^{\circ} \mathrm{C}$ were the optimal conditions, affording alkenylated product 11 in $69 \%$ isolated yield with exclusive $E$ selectivity (Table 1, entry 1). Reducing the amount of styrene from 10 equiv. to 5 equiv. resulted in a much lower yield (Table 1, entry 2). After careful analysis of the crude product mixture using GC-MS and ${ }^{1} \mathrm{H}$ NMR spectroscopy (see Supplementary Discussion), it was found that cobalt hydride, a key intermediate in the proposed catalytic cycle (Fig. 2), also promoted the oligomerization of styrene ${ }^{53}$. Due to this competing side reaction, 10 equiv of styrene is needed to achieve a high yield of the desired product. Nonetheless, considering the abundance of alkene feedstocks ( $\$ 0.002 / \mathrm{mmol}$ for styrene) and the versatile reactivity of olefins, this method is still highly valuable. Hydrogen gas was produced in $64 \%$ yield by GC analysis of the crude product mixture (Supplementary Figs. 3-5), which supported our mechanistic hypothesis. The possibility of styrene or styrene oligomers serving as hydrogen acceptors ${ }^{54}$ was excluded since byproducts from olefin hydrogenation were not detected (Supplementary Figs. 6-9). During the reaction optimization, we found that the ligand had a large impact on the dehydrogenative alkenylation (Table 1, entries 3-12). Axial ligands on cobaloxime can readily dissociate, and recoordination of these ligands facilitates $\beta$ - $\mathrm{H}$ elimination ${ }^{55,56}$. Moreover, the electronic and steric properties of axial ligands could dramatically influence the reactivity of cobaloxime complexes ${ }^{56,57}$. A survey of heterocyclic ligands (Supplementary Table 8) revealed that 2,6-lutidine (L5) was most effective among all the pyridine ligands evaluated, most probably because it provided a suitable steric environment around the cobalt center. In addition, the use of $50 \mathrm{~mol} \%$ of 2 methylbenzimidazole (L9) was also effective. The use of inorganic bases such as sodium bicarbonate failed to increase reaction yield, confirming that these heterocycles function as ligands rather than bases (Table 1, entry 13 and Supplementary Table 9). No product was detected in the absence of TBADT $\mathbf{9}$, cobaloxime $\mathbf{1 0}$ or light (Table 1, entry 14), demonstrating that all these components are required. It was noted that other direct HAT photocatalysts resulted in very low conversions under the established conditions (Table 1, entry 15).

Substrate scope. With the optimized conditions in hand, we set out to examine the scope and site-selectivity of this $\mathrm{C}-\mathrm{H}$ alkenylation (Fig. 3). Throughout these studies, the $\mathrm{C}-\mathrm{H}$ substrate was used as the limiting reagent. A wide range of alkanes were smoothly alkenylated in moderate to good yields with exclusive $E$ selectivity (11-32). Cyclic alkanes with ring sizes ranging from 5 
Table 1 Selected optimization results.

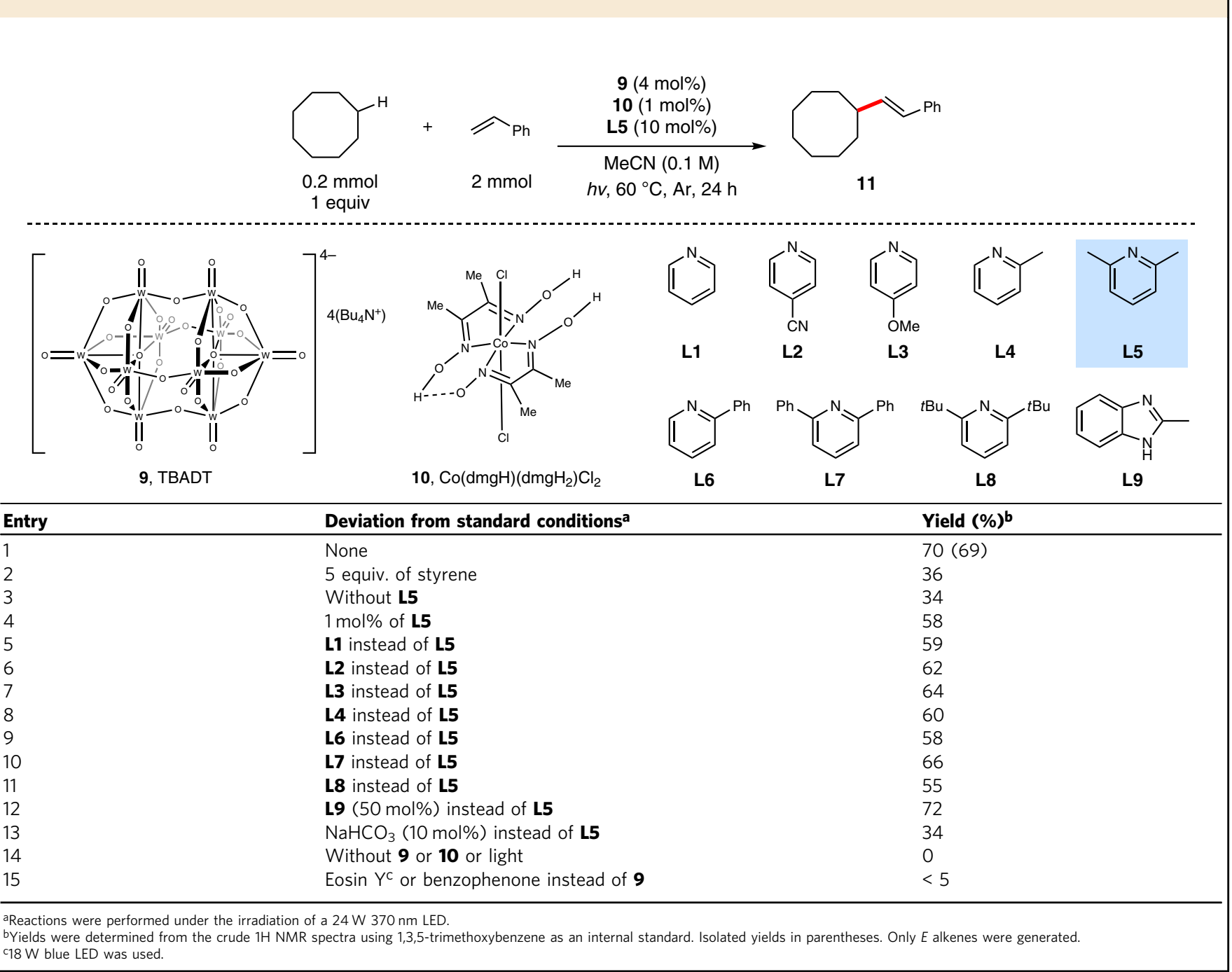

to 12 gave alkenylated products 11-15 in good yields (54-78\%). The alkenylation reaction was amenable to scale-up, delivering alkene 16 in $71 \%$ yield on a $5.0-\mathrm{mmol}$ scale. Interestingly, this dual-catalytic system exhibits a strong preference for the functionalization of sterically accessible and electron-rich $\mathrm{C}-\mathrm{H}$ sites. For instance, the secondary $\mathrm{C}-\mathrm{H}$ bonds in norbornane (16), 1,4epoxycyclohexane (17) and trans-1,4-dimethylcyclohexane (18) were selectively alkenylated, while the sterically hindered tertiary $\mathrm{C}-\mathrm{H}$ bonds were not reactive despite their relatively lower bond dissociation energies (BDEs) ${ }^{58}$. Primary $\mathrm{C}-\mathrm{H}$ bonds, owing to their high $\mathrm{BDEs}^{58}$, were barely functionalized in the presence of secondary $\mathrm{C}-\mathrm{H}$ bonds (18-19). The steric preference of this transformation is also illustrated by the regiospecific alkenylation of 1-methylcyclopentanol (19) at the remote methylene site. Similarly, the least hindered and most electron-rich methylene site of azaspirodecanedione (20) was selectively alkenylated. The reaction of adamantane (21) furnished two regioisomers (1.3:1) in $82 \%$ yield, while the reaction of adamantane derivatives bearing electron-withdrawing groups (22-24) occurred predominantly at the tertiary sites. This unique selectivity observed among adamantane derivatives probably originated from the steric accessibility of the tertiary $\mathrm{C}-\mathrm{H}$ bond due to its equatorial character ${ }^{59}$ and the remarkably long life-time of 1-adamantyl radicals compared to 2-adamantyl radicals ${ }^{60}$. For linear alkanes, pentane (25, $\alpha: \beta: \gamma 1.1: 4.2: 1)$ and hexane (26, $\alpha: \beta: \gamma 1: 3.7: 1.7)$ were preferentially functionalized at the internal secondary positions. Hexamethyldisilane, which possesses one of the strongest $\mathrm{C}_{\mathrm{sp} 3}-\mathrm{H}$ bonds $(\mathrm{BDE}=101.3 \mathrm{kcal} / \mathrm{mol}$, see Supplementary Discussion), underwent alkenylation to afford product 27 in $50 \%$ yield. Activated $\mathrm{C}_{\mathrm{sp} 3}-\mathrm{H}$ bonds were also examined. Ethers (28-30), amides (31) and alkylbenzenes (32) were all found to be competent substrates (38-67\%). Notably, substituted tetrahydropyrans afforded alkenylation products (29 and 30) with excellent regio- and diastereoselectivity.

$\alpha, \beta$-Unsaturated ketones are core structures in a wide variety of naturally occurring and man-made molecules that are of synthetic, biological and pharmaceutical importance ${ }^{61}$. The direct alkenylation of aldehydes represents a highly attractive and sustainable strategy for the synthesis of this important family of compounds. We were excited to find that various primary and secondary aldehydes were effective substrates under the optimal alkenylation conditions, providing $\alpha, \beta$-unsaturated ketones in decent yields with exclusive $E$ selectivity (33-43). Notably, excellent site selectivity was observed for aldehyde $\mathrm{C}-\mathrm{H}$ bonds, while other activated $\mathrm{C}-\mathrm{H}$ bonds, such as benzylic (34), allylic (38), propargylic (39) and $\alpha$-heteroatom $\mathrm{C}-\mathrm{H}$ bonds (42 and 43), were not functionalized. The preference of formyl $\mathrm{C}-\mathrm{H}$ abstraction by decatungstate anion can be explained by the polar transition state due to the partial positive charge on the carbonyl carbon (Supplementary Fig. 21) ${ }^{62}$. A tertiary aldehyde (44), 2- 

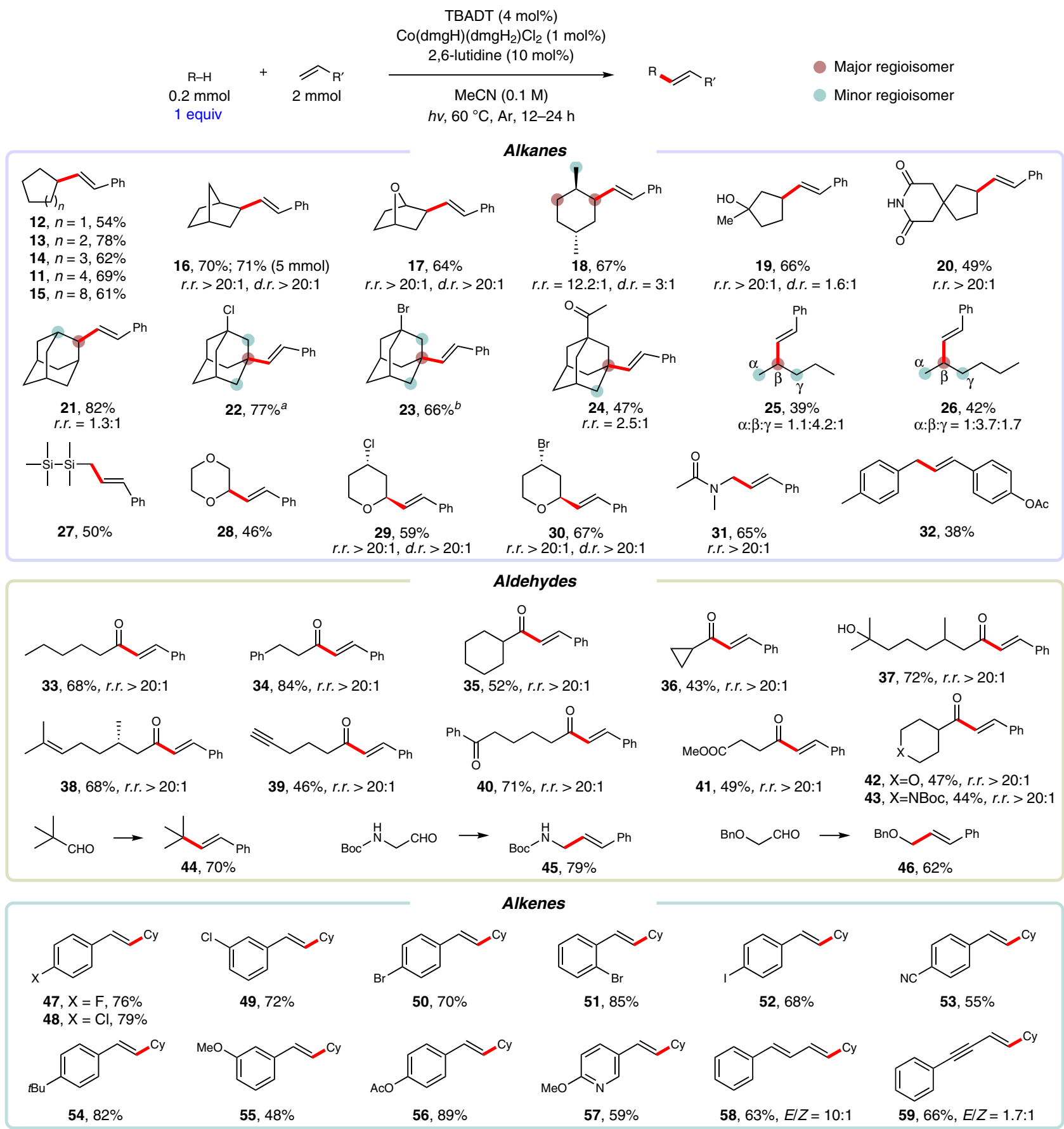

Fig. 3 Scope of dehydrogenative alkenylation. Isolated yields. $E / Z>99: 1$ unless otherwise noted. ${ }^{a} 60 \%$ selectivity. ${ }^{b} 67 \%$ selectivity. Ac $=$ acetyl, Boc $=$ tert-butyloxycarbonyl, $\mathrm{Bn}=$ benzyl, $\mathrm{Cy}=$ cyclohexyl.

tert-butyloxycarbonylamino and 2-benzyloxy acetaldehydes (45 and 46) acted as alkyl radical equivalents and gave decarbonylative alkenylated products exclusively in high yields $(62-79 \%)$. However, aromatic aldehydes gave poor yields under the established reaction conditions.

The generality of this $\mathrm{C}-\mathrm{H}$ alkenylation with respect to the alkene component was subsequently investigated. Electrondeficient (47-53) and electron-rich (54-56) styrene derivatives possessing ortho-, meta-, or para-substituents were all found to efficiently provide the alkenylated products in moderate to good yields and with exclusive $E$ selectivity. The 1,2-disubstituted vinyl pyridine product (57) could be obtained in 59\% yield. 1,3-Dienes (58) and enynes (59) were also suitable coupling partners, even though the $E / Z$ selectivity was diminished. Importantly, functional groups that are typically sensitive to transition-metal catalysis, such as alkyl bromides (23 and 30), alkenes (38), alkynes (39) and aryl iodides (52), were all well tolerated, allowing for subsequent orthogonal functionalization reactions.

Late-stage selective $\mathrm{C}-\mathrm{H}$ alkenylation of complex molecules. The potential of this protocol for the late-stage site-selective alkenylation of complex molecules was subsequently investigated. As illustrated in Fig. 4, a number of natural products and derivatives were successfully alkenylated under the optimal conditions. The observed regioselectivities were in accordance with the 


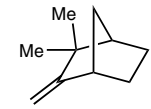

\section{( \pm )-Camphene} 1 equiv

$16 \mathrm{C}-\mathrm{H}$ bonds

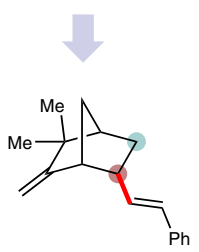

$60,57 \%, r . r=1.4: 1$ d. $r>20: 1, E / Z>99: 1$

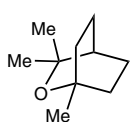

Eucalyptol

$18 \mathrm{C}-\mathrm{H}$ bonds

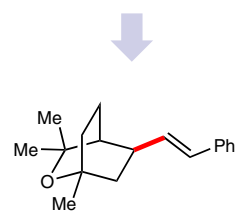

$61,51 \%, r . r>20: 1$ d. $r>20: 1, E / Z>99: 1$

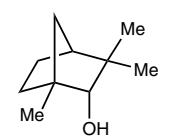

(+)-Fenchol 1 equiv

$17 \mathrm{C}-\mathrm{H}$ bonds

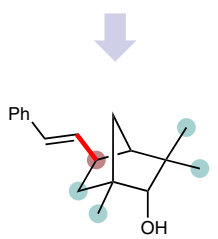

62, $46 \%^{a}, 74 \%$ selectivity d. $r>20: 1, E / Z>99: 1$

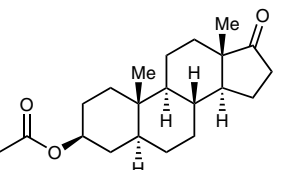

trans-Androsterone acetate 1 equiv

$32 \mathrm{C}-\mathrm{H}$ bonds

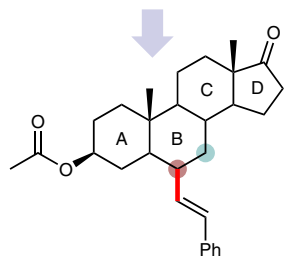

63, $64 \%, r . r=2.5: 1$ d. $r>20: 1, E / Z>99: 1$

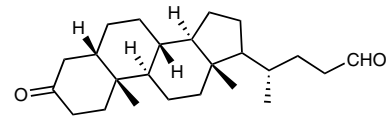

Lithocholic acid derivative 1 equiv $38 \mathrm{C}-\mathrm{H}$ bonds

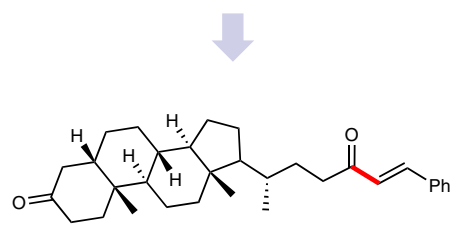

64, $75 \%$ r.r $>20: 1, E / Z>99: 1$

Fig. 4 Late-stage alkenylation of natural products and derivatives. Isolated yields. Standard conditions unless otherwise noted. ${ }^{a}$ Overall yields over three steps: (1) protection: hexamethyldisilazane (0.6 equiv.), ammonium thiocyanate ( 0.05 equiv.), DCM, $0^{\circ} \mathrm{C} \rightarrow \mathrm{RT}$, $12 \mathrm{~h}$; (2) alkenylation: standard conditions for dehydrogenative alkenylation; (3) deprotection: tetra- $n$-butylammonium fluoride (1 equiv.), THF, RT, 12 h. DCM = dichloromethane. THF $=$ tetrahydrofuran.

preferences described above. Camphene, a terminal-olefincontaining natural product, was alkenylated at sterically accessible methylene sites $(\mathbf{6 0}, 57 \%)$. A moderate yield of alkenylated eucalyptol was obtained $(\mathbf{6 1}, 51 \%)$ with excellent site selectivity (over 20 to 1 ) for the less hindered methylene site. This selectivity is remarkable, especially compared to TBADT-catalyzed photooxygenation of eucalyptol (2.4 to 1 methylene selectivity $)^{63}$. Useful efficiencies were observed for the terpenoid (+)-fenchol with $74 \%$ regioselectivity for the unactivated and less hindered methylene sites $(62,46 \%)$. The steroid trans-androsterone acetate, which contains five tertiary $\mathrm{C}-\mathrm{H}$ bonds and nine methylene sites, underwent selective alkenylation on the $\mathrm{B}$ ring $(63,64 \%)$. This selectivity is predictable, as the $\mathrm{C}-\mathrm{H}$ bonds on the A ring and $\mathrm{D}$ ring are electronically deactivated, while the $\mathrm{C}-\mathrm{H}$ bonds on the $\mathrm{C}$ ring are sterically inaccessible. Finally, a complex aldehyde derivative of lithocholic acid was readily functionalized, delivering $\alpha, \beta$ unsaturated ketone $\mathbf{6 4}$ in $75 \%$ yield with excellent selectivity.

Mechanistic considerations. Although excited decatungstate anions have been applied to abstract electron-rich and sterically accessible $\mathrm{C}-\mathrm{H}$ bonds ${ }^{64}$, the excellent sterically controlled selectivity of this $\mathrm{C}-\mathrm{H}$ alkenylation (e.g., eucalyptol 61) prompted us to investigate the potential role of the cobaloxime catalysts. Control experiments with different ligands on the cobalt catalyst (Supplementary Table 12) suggested that planar dimethylglyoximate ligands gave the cobalt catalyst exceptional steric bulk ${ }^{65}$ and contributed to the high regioselectivity. In addition, various control experiments have been conducted to further support the proposed mechanism in Fig. 2 (see Supplementary Discussion). The presence of radical intermediates was evidenced by radical trapping experiments using 2,2,6,6-tetramethylpiperidine- $N$-oxyl (TEMPO, Supplementary Fig. 10). Light on-off experiments demonstrated that continuous light irradiation was necessary for the transformation (Supplementary Fig. 13). We measured the kinetic isotope effect (KIE) from two parallel reactions and an intermolecular competition experiment, and the KIE values were calculated to be 1.0 and 2.0 respectively (Supplementary Figs. 14-16). These results indicated that $\mathrm{C}-\mathrm{H}$ cleavage may not be the rate-determining step $^{66}$. The UV-Vis absorption of the reaction mixture was also monitored. Two absorption bands at 440-500 nm and 550-700 nm were observed after $10 \mathrm{~min}$ light irradiation and subsequent exposure to air (Supplementary Figs. 17-18), which indicates the presence of $\mathrm{Co}^{\mathrm{II}}$ and $\mathrm{Co}^{\mathrm{I}}$ intermediates respectively ${ }^{48,49}$.

\section{Discussion}

In summary, we disclosed a general strategy for the dehydrogenative alkenylation of alkanes and aliphatic aldehydes with aryl alkenes using the $\mathrm{C}-\mathrm{H}$ substrates as the limiting reagent. The late-stage functionalization of complex pharmaceutically important molecules was achieved with high levels of site selectivity, and a broad scope of functional groups were tolerated, providing a unique method for olefin synthesis and molecular diversification. The sterically and electronically dictated site selectivity originates from the dual effect of both the decatungstate and cobaloxime catalysts. Our future efforts will be directed toward further expanding the alkene scope and developing strategies to further tune the site-selectivity of the $\mathrm{C}-\mathrm{H}$ alkenylation.

\section{Methods}

General procedure for dehydrogenative alkenylation of alkanes and aldehydes with alkenes. To a $10 \mathrm{~mL}$ oven-dried Schlenk tube equipped with a magnetic stir bar was added the corresponding $\mathrm{C}-\mathrm{H}$ nucleophile $(0.2 \mathrm{mmol}, 1.0$ equiv. $)$, alkene ( $2.0 \mathrm{mmol}, 10$ equiv.), TBADT ( $26.6 \mathrm{mg}, 0.008 \mathrm{mmol}, 4 \mathrm{~mol} \%), \mathrm{Co}(\mathrm{dmgH})$ $\left(\mathrm{dmgH}_{2}\right) \mathrm{Cl}_{2}(0.7 \mathrm{mg}, 0.002 \mathrm{mmol}, 1 \mathrm{~mol} \%), 2,6$-lutidine $(2.1 \mathrm{mg}, 0.02 \mathrm{mmol}, 10 \mathrm{~mol}$ $\%)$ and dry acetonitrile $(2 \mathrm{~mL})$. The resulting mixture was cooled to $0^{\circ} \mathrm{C}$ using an ice-water bath, and bubbled with argon balloon for $10 \mathrm{~min}$ (if the $\mathrm{C}-\mathrm{H}$ nucleophile was volatile, it was added after argon bubbling). After that, the reaction was placed under a $370 \mathrm{~nm}$ LED (2.5 meter strips, $24 \mathrm{~W})$, stirred and irradiated under argon atmosphere. The temperature was maintained at $60^{\circ} \mathrm{C}$ using a water bath. The reaction mixture was removed from light and quenched by stirring open to air for 5 minutes. The solvent was removed on a rotary evaporator under reduced pressure and the residue was subjected to column chromatography isolation over silica gel or preparative thin layer chromatography to obtain the corresponding product.

\section{Data availability}

The authors declare that all other data supporting the findings of this study are available within the article and Supplementary Information files, and also are available from the corresponding author upon reasonable request.

Received: 11 February 2020; Accepted: 25 March 2020;

Published online: 23 April 2020

\section{References}

1. Smith, M. B. \& March, J. March's Advanced Organic Chemistry: Reactions, Mechanisms, and Structure 6th edn. (John Wiley \& Sons, Inc., 2007).

2. Le Bras, J. \& Muzart, J. Intermolecular dehydrogenative Heck reactions. Chem. Rev. 111, 1170-1214 (2011).

3. Yamaguchi, J., Yamaguchi, A. D. \& Itami, K. C-H bond functionalization: emerging synthetic tools for natural products and pharmaceuticals. Angew. Chem. Int. Ed. 51, 8960-9009 (2012). 
4. Fujiwara, Y., Moritani, I., Danno, S., Asano, R. \& Teranishi, S. Aromatic substitution of olefins. VI. Arylation of olefins with palladium(II) acetate. $J$. Am. Chem. Soc. 91, 7166-7169 (1969).

5. Jia, C., Kitamura, T. \& Fujiwara, Y. Catalytic functionalization of arenes and alkanes via C-H bond activation. Acc. Chem. Res. 34, 633-639 (2001).

6. Zhou, L. \& Lu, W. Towards ideal synthesis: alkenylation of aryl C-H bonds by a Fujiwara-Moritani reaction. Chem. Eur. J. 20, 634-642 (2014)

7. Wasa, M., Engle, K. M. \& Yu, J.-Q. Pd(II)-catalyzed olefination of $s p^{3} \mathrm{C}-\mathrm{H}$ bonds. J. Am. Chem. Soc. 132, 3680-3681 (2010).

8. Stowers, K. J., Fortner, K. C. \& Sanford, M. S. Aerobic Pd-catalyzed $\mathrm{sp}^{3}$ C-H olefination: a route to both N-heterocyclic scaffolds and alkenes. J. Am. Chem. Soc. 133, 6541-6544 (2011).

9. Li, S., Chen, G., Feng, C.-G., Gong, W. \& Yu, J.-Q. Ligand-enabled $\gamma-C-H$ olefination and carbonylation: construction of $\beta$-quaternary carbon centers. J. Am. Chem. Soc. 136, 5267-5270 (2014).

10. He, J. et al. Ligand-controlled $\mathrm{C}\left(\mathrm{sp}^{3}\right)-\mathrm{H}$ arylation and olefination in synthesis of unnatural chiral $\alpha$-amino acids. Science 343, 1216-1220 (2014).

11. Jiang, H., He, J., Liu, T. \& Yu, J.-Q. Ligand-enabled $\gamma$-C $\left(\mathrm{sp}^{3}\right)$-H olefination of amines: en route to pyrrolidines. J. Am. Chem. Soc. 138, 2055-2059 (2016).

12. DeBoef, B., Pastine, S. J. \& Sames, D. Cross-coupling of $\mathrm{sp}^{3} \mathrm{C}-\mathrm{H}$ bonds and alkenes: catalytic cyclization of alkene-amide substrates. J. Am. Chem. Soc. 126, 6556-6557 (2004).

13. Shi, Z., Schröder, N. \& Glorius, F. Rhodium(III)-catalyzed dehydrogenative Heck reaction of salicylaldehydes. Angew. Chem. Int. Ed. 51, 8092-8096 (2012).

14. Chuentragool, P. et al. Aliphatic radical relay Heck reaction at unactivated C $\left(\mathrm{sp}^{3}\right)-\mathrm{H}$ sites of alcohols. Angew. Chem. Int. Ed. 58, 1794-1798 (2019).

15. Zhu, Y. \& Wei, Y. Copper catalyzed direct alkenylation of simple alkanes with styrenes. Chem. Sci. 5, 2379-2382 (2014).

16. Liu, D., Liu, C., Li, H. \& Lei, A. Copper-catalysed oxidative C-H/C-H coupling between olefins and simple ethers. Chem. Commun. 50, 3623-3626 (2014).

17. $\mathrm{Gu}, \mathrm{H}$. \& Wang, C. Rhenium-catalyzed dehydrogenative olefination of $\mathrm{C}\left(\mathrm{sp}^{3}\right)$ $\mathrm{H}$ bonds with hypervalent iodine(III) reagents. Org. Biomol. Chem. 13 5880-5884 (2015).

18. Wang, J., Liu, C., Yuan, J. \& Lei, A. Copper-catalyzed oxidative coupling of alkenes with aldehydes: direct access to $\alpha, \beta$-unsaturated ketones. Angew. Chem. Int. Ed. 52, 2256-2259 (2013).

19. Song, C.-X. et al. Direct functionalization of benzylic C-Hs with vinyl acetates via Fe-catalysis. Chem. Commun. 40, 6002-6004 (2009).

20. Li, Z., Bohle, D. S. \& Li, C. Cu-catalyzed cross-dehydrogenative coupling: a versatile strategy for $\mathrm{C}-\mathrm{C}$ bond formations via the oxidative activation of $\mathrm{sp}^{3}$ C-H bonds. Proc. Natl Acad. Sci. USA 103, 8928-8933 (2006).

21. Jazzar, R. et al. Functionalization of organic molecules by transition-metalcatalyzed C( $\left(\mathrm{sp}^{3}\right)-\mathrm{H}$ activation. Chem. Eur. J. 16, 2654-2672 (2010).

22. Patai, S. The Chemistry of the Metal-Carbon Bond (ed. Stille, J. K.) Vol. 2 (Wiley: New York, 1985)

23. Garralda, M. A. Aldehyde C-H activation with late transition metal organometallic compounds. Formation and reactivity of acyl hydrido complexes. Dalton Trans. 3635-3645 (2009).

24. Newhouse, T. \& Baran, P. S. If C-H bonds could talk: selective C-H bond oxidation. Angew. Chem. Int. Ed. 50, 3362-3374 (2011).

25. Xue, F. et al. Reaction discovery using acetylene gas as the chemical feedstock accelerated by the "stop-flow" micro-tubing reactor system. Chem. Sci. 8, 3623-3627 (2017).

26. Deng, H.-P. et al. Photoinduced nickel-catalyzed chemo- and regioselective hydroalkylation of internal alkynes with ether and amide $\alpha$-hetero $\mathrm{C}\left(\mathrm{sp}^{3}\right)-\mathrm{H}$ bonds. J. Am. Chem. Soc. 139, 13579-13584 (2017).

27. Cao, H. et al. Photo-induced decarboxylative Heck-type coupling of unactivated aliphatic acids and terminal alkenes in the absence of sacrificial hydrogen acceptors. J. Am. Chem. Soc. 140, 16360-16367 (2018).

28. Zhou, R., Liu, H., Tao, H., Yu, X. \& Wu, J. Metal-free direct alkylation of unfunctionalized allylic/benzylic $\mathrm{sp}^{3} \mathrm{C}-\mathrm{H}$ bonds via photoredox induced radical cation deprotonation. Chem. Sci. 8, 4654-4659 (2017).

29. Fan, X.-Z. et al. Eosin $\mathrm{Y}$ as a direct hydrogen-atom transfer photocatalyst for the functionalization of C-H bonds. Angew. Chem. Int. Ed. 57, 8514-8518 (2018).

30. Deng, H.-P., Zhou, Q. \& Wu, J. Microtubing-reactor-assisted aliphatic C-H functionalization with $\mathrm{HCl}$ as a hydrogen-atom-transfer catalyst precursor in conjunction with an organic photoredox catalyst. Angew. Chem. Int. Ed. 57, 12661-12665 (2018).

31. Kuang, Y. et al. Asymmetric synthesis of 1,4-dicarbonyl compounds from aldehydes by hydrogen atom transfer photocatalysis and chiral Lewis acid catalysis. Angew. Chem. Int. Ed. 58, 16859-16863 (2019).

32. Cernak, T. et al. The medicinal chemist's toolbox for late stage functionalization of drug-like molecules. Chem. Soc. Rev. 45, 546-576 (2016).

33. Capaldo, L. \& Ravelli, D. Hydrogen atom transfer (HAT): a versatile strategy for substrate activation in photocatalyzed organic synthesis. Eur. J. Org. Chem. 2056-2071 (2017).
34. Tzirakis, M. D., Lykakis, I. N. \& Orfanopoulos, M. Decatungstate as an efficient photocatalyst in organic chemistry. Chem. Soc. Rev. 38, 2609-2621 (2009).

35. Ravelli, D., Protti, S. \& Fagnoni, M. Decatungstate anion for photocatalyzed "window ledge" reactions. Acc. Chem. Res. 49, 2232-2242 (2016).

36. West, J. G., Huang, D. \& Sorensen, E. J. Acceptorless dehydrogenation of small molecules through cooperative base metal catalysis. Nat. Commun. 6, 10093 (2015).

37. Halperin, S. D. et al. Convenient photocatalytic fluorination of unactivated C$\mathrm{H}$ bonds. Angew. Chem. Int. Ed. 53, 4690-4693 (2014).

38. Perry, I. B. et al. Direct arylation of strong aliphatic C-H bonds. Nature 560 , 70-75 (2018)

39. Esposti, S., Dondi, D., Fagnoni, M. \& Albini, A. Acylation of electrophilic olefins through decatungstate-photocatalyzed activation of aldehydes. Angew. Chem. Int. Ed. 46, 2531-2534 (2007).

40. Chen, B., Wu, L.-Z. \& Tung, C.-H. Photocatalytic activation of less reactive bonds and their functionalization via hydrogen-evolution cross-couplings. Acc. Chem. Res. 51, 2512-2523 (2018).

41. Tang, S., Zeng, L. \& Lei, A. Oxidative $\mathrm{R}^{1}-\mathrm{H} / \mathrm{R}^{2}-\mathrm{H}$ cross-coupling with hydrogen evolution. J. Am. Chem. Soc. 140, 13128-13135 (2018).

42. Wang, H., Gao, X., Lv, Z., Abdelilah, T. \& Lei, A. Recent advances in oxidative $\mathrm{R}^{1}-\mathrm{H} / \mathrm{R}^{2}-\mathrm{H}$ cross-coupling with hydrogen evolution via photo-/ electrochemistry. Chem. Rev. 119, 6769-6787 (2019).

43. Yu, W.-L. et al. Dehydrogenative silylation of alkenes for the synthesis of substituted allylsilanes by photoredox, hydrogen-atom transfer, and cobalt catalysis. Angew. Chem. Int. Ed. 58, 10941-10945 (2019).

44. Tian, W.-F. et al. Visible-light photoredox-catalyzed decarboxylative alkylation of heteroarenes using carboxylic acids with hydrogen release. Org. Lett. 21, 6930-6935 (2019).

45. De Waele, V., Poizat, O., Fagnoni, M., Bagno, A. \& Ravelli, D. Unraveling the key features of the reactive state of decatungstate anion in hydrogen atom transfer (HAT) photocatalysis. ACS Catal. 6, 7174-7182 (2016).

46. Branchaud, B. P. \& Choi, Y. L. Effect of a remote ligand substituent on premature $\beta-\mathrm{H}$ elimination in a cobaloxime-mediated radical alkyl-alkenyl cross coupling. Tetrahedron Lett. 29, 6037-6038 (1988)

47. Garr, C. D. \& Finke, R. G. Radical cage effects in adocobinamide (axial-baseoff coenzyme $\mathrm{B}_{12}$ ): A simple method for trapping [Ado ${ }^{\circ} \mathrm{Co}^{\mathrm{II}}$ ] radical pairs, a new $\beta$-H elimination product from the radical pair and measurement of an unprecedentedly large cage-recombination efficiency factor, $\mathrm{F}_{\mathrm{c}} \geq 0.94$. J. Am Chem. Soc. 114, 10440-10445 (1992).

48. Du, P., Knowles, K. \& Eisenberg, R. A homogeneous system for the photogeneration of hydrogen from water based on a platinum(II) terpyridyl acetylide chromophore and a molecular cobalt catalyst. J. Am. Chem. Soc. 130, 12576-12577 (2008)

49. Lazarides, T. et al. Making hydrogen from water using a homogeneous system without noble metals. J. Am. Chem. Soc. 131, 9192-9194 (2009).

50. Dempsey, J. L., Brunschwig, B. S., Winkler, J. R. \& Gray, H. B. Hydrogen evolution catalyzed by cobaloximes. Acc. Chem. Res. 42, 1995-2004 (2009).

51. Du, P. et al. Visible light-driven hydrogen production from water catalyzed by molecular cobaloxime catalysts. Inorg. Chem. 48, 4952-4962 (2009).

52. Yamase, T., Takabayashi, N. \& Kaji, M. Solution photochemistry of tetrakis (tetrabutylammonium) decatungstate(VI) and catalytic hydrogen evolution from alcohols. J. Chem. Soc., Dalton Trans. 793-799 (1984).

53. Debuigne, A. et al. Overview of cobalt-mediated radical polymerization: Roots state of the art and future prospects. Prog. Polym. Sci. 34, 211-239 (2009).

54. Wang, G.-Z., Shang, R. \& Fu, Y. Irradiation-induced palladium-catalyzed decarboxylative Heck reaction of aliphatic $N$-(acyloxy)phthalimides at room temperature. Org. Lett. 20, 888-891 (2018).

55. Schrauzer, G. N. \& Grate, J. H. Sterically induced, spontaneous Co-C bond homolysis and $\beta$-elimination reactions of primary and secondary organocobalamins. J. Am. Chem. Soc. 103, 541-546 (1981).

56. Schrauzer, G. N., Lee, L.-P. \& Sibert, J. W. Alkylcobalamins and alkylcobaloximes. Electronic structure, spectra, and mechanism of photodealkylation. J. Am. Chem. Soc. 92, 2997-3005 (1970)

57. Panagiotopoulos, A., Ladomenou, K., Sun, D., Artero, V. \& Coutsolelos, A. G. Photochemical hydrogen production and cobaloximes: the influence of the cobalt axial N-ligand on the system stability. Dalton Trans. 45, 6732-6738 (2016).

58. Luo, Y.-R. Comprehensive Handbook of Chemical Bond Energies (CRC Press: Boca Raton, FL, 2007)

59. Tabushi, I., Hamuro, J. \& Oda, R. Free-radical substitution on adamantane. J. Am. Chem. Soc. 89, 7127-7129 (1967).

60. Tabushi, I., Aoyama, Y., Kojo, S., Hamuro, J. \& Yoshida, Z. Free-radical halogenation of adamantane. Selectivity and relative lifetime of 1-and 2adamantyl radicals. J. Am. Chem. Soc. 94, 1177-1183 (1972).

61. Tanaka, T., Kawase, M. \& Tani, S. Urease inhibitory activity of simple $\alpha, \beta-$ unsaturated ketones. Life Sci. 73, 2985-2990 (2003).

62. Roberts, B. P. Polarity-reversal catalysis of hydrogen-atom abstraction reactions: concepts and applications in organic chemistry. Chem. Soc. Rev. 28, 25-35 (1999) 
63. Zakrzewski, J. \& Giannotti, C. Photo-oxygenation of 1,8-cineole by molecular oxygen catalysed by $\left(\mathrm{Bu}_{4} \mathrm{~N}\right)_{4} \mathrm{~W}_{10} \mathrm{O}_{32}$. J. Photochem. Photobiol. A: Chem. 63 , 173-177 (1992).

64. Ravelli, D. et al. Site-selective C-H functionalization by decatungstate anion photocatalysis: Synergistic control by polar and steric effects expands the reaction scope. ACS Catal. 8, 701-713 (2018).

65. Nishikubo, Y. \& Branchaud, B. P. Cobaloxime $\pi$-cation steric and stereoelectronic effects: The amazing effect of a single methyl group adjacent to the site of reaction. J. Am. Chem. Soc. 121, 10924-10927 (1999).

66. Simmons, E. M. \& Hartwig, J. F. On the interpretation of deuterium kinetic isotope effects in $\mathrm{C}-\mathrm{H}$ bond functionalizations by transition-metal complexes. Angew. Chem. Int. Ed. 51, 3066-3072 (2012).

\section{Acknowledgements}

We are grateful for the financial support provided by the National University of Singapore and the Ministry of Education (MOE) of Singapore (MOE2017-T2-2-081), NUS (Suzhou) Research Institute and National Natural Science Foundation of China (Grant No. 21702142, 21771135, 21871205). We thank Mr. Tang Jie (National University of Singapore) for the help on quantitative analysis of generated hydrogen gas.

\section{Author contributions}

H.C. discovered and developed the reaction. H.C., Y.K., and J.W. conceived and designed the investigations. X.S. conducted density functional theory (DFT) calculations. H.C., K.L.W., B.B.T., and J.M.C.K. performed the experiments. H.C., X.L., and J.W. wrote the manuscript.

\section{Competing interests}

The authors declare no competing interests.

\section{Additional information}

Supplementary information is available for this paper at https://doi.org/10.1038/s41467020-15878-6.

Correspondence and requests for materials should be addressed to J.W.

Peer review information Nature Communications thanks Yang Li and the other, anonymous, reviewer(s) for their contribution to the peer review of this work. Peer reviewer reports are available.

Reprints and permission information is available at http://www.nature.com/reprints

Publisher's note Springer Nature remains neutral with regard to jurisdictional claims in published maps and institutional affiliations.

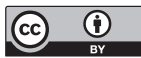

Open Access This article is licensed under a Creative Commons Attribution 4.0 International License, which permits use, sharing, adaptation, distribution and reproduction in any medium or format, as long as you give appropriate credit to the original author(s) and the source, provide a link to the Creative Commons license, and indicate if changes were made. The images or other third party material in this article are included in the article's Creative Commons license, unless indicated otherwise in a credit line to the material. If material is not included in the article's Creative Commons license and your intended use is not permitted by statutory regulation or exceeds the permitted use, you will need to obtain permission directly from the copyright holder. To view a copy of this license, visit http://creativecommons.org/ licenses/by/4.0/.

(C) The Author(s) 2020 